\title{
Preparation and Characterization of Novel Electrospinnable PBT/POSS Hybrid Systems Starting from c-PBT
}

\author{
Lorenza Gardella, ${ }^{1}$ Alberto Fina, ${ }^{2}$ and Orietta Monticelli ${ }^{1}$ \\ ${ }^{1}$ Dipartimento di Chimica e Chimica Industriale, Università di Genova, Via Dodecaneso 31, 16146 Genova, Italy \\ ${ }^{2}$ Dipartimento di Scienza Applicata e Tecnologia, Politecnico di Torino-Sede di Alessandria, Viale Teresa Michel 5, \\ 15121 Alessandria, Italy
}

Correspondence should be addressed to Orietta Monticelli; orietta.monticelli@unige.it

Received 10 December 2014; Revised 7 April 2015; Accepted 9 April 2015

Academic Editor: Ashok K. Sundramoorthy

Copyright (C) 2015 Lorenza Gardella et al. This is an open access article distributed under the Creative Commons Attribution License, which permits unrestricted use, distribution, and reproduction in any medium, provided the original work is properly cited.

Novel hybrid systems based on poly(butyleneterephthalate) (PBT) and polyhedral oligomeric silsesquioxanes (POSS) have been prepared by applying the ring-opening polymerization of cyclic poly(butyleneterephthalate) oligomers. Two types of POSS have been used: one characterized by hydroxyl functionalities (named POSS-OH) and another without specific reactive groups (named oib-POSS). It was demonstrated that POSS-OH acts as an initiator for the polymerization reaction, leading to the direct insertion of the silsesquioxane into the polymer backbone. Among the possible applications of the PBT/POSS hybrid system, the possibility to obtain nanofibers has been assessed in this work.

\section{Introduction}

Polyhedral oligomeric silsesquioxanes (POSS) are ideal nanobuilding blocks for the synthesis of organic-inorganic hybrid materials [1-3]. Indeed, incorporation of POSS into polymer matrices results in improvement of properties, such as mechanical properties [4], thermal stability [5-7], flammability [8], gas permeability, and dielectric permittivity [9].

Recently, POSS-based polymeric materials have been produced also by using electrospinning, a simple and effective technique to generate continuous fibers ranging from micrometer to nanometer size in diameter [10]. Two different approaches have been proposed for the preparation of these nanofibers. POSS can be added directly into the polymer electrospinning solution. By this method, several polymers, such as cellulose acetate [11], polyvinylidene fluoride [12], sulfonated poly(arylene ether sulfone) [13], poly(vinyl alcohol) [14], poly(N-isopropylacrylamide) [15], poly(butylene terephthalate) (PBT) [16], ethylene-propylene-diene rubber [17], poly(styrene-co-maleic anhydride) [18], polylactic acid $[19,20]$, and protein $[21]$, were exploited to prepare electrospun POSS-based nanofibers.
Nevertheless, the method, which involves the direct solubilization of POSS in the electrospinning solution, could be challenging because of the need to find a common solvent for the silsesquioxane and the polymer and because of the need to limit POSS concentration in the solution in order to obtain its nanometric distribution. Few papers report on the second method, which consists in the electrospinning of hybrids, namely, systems containing silsesquioxane molecules chemically bound to the macromolecule backbone. Kim et al. [22] studied the water resistance of the nanofiber web, obtained by electrospinning of a hybrid PVA/POSS, and incorporating silsesquioxane molecules into hydrophilic PVA backbone. $\mathrm{Wu}$ et al. [23] reported on the preparation of nanostructured fibrous hydrogel scaffolds of POSS-poly(ethylene glycol) hybrid thermoplastic polyurethanes incorporating $\mathrm{Ag}$ for antimicrobial applications whereas superhydrophobic electrospun fibers based on POSS-polymethylmethacrylate copolymers were prepared by Xue et al. [24]. Despite the limited efforts in this field, this kind of processing opens up POSS-based hybrids for interesting applications in several fields such as biomedicine and filtration. A second motivation for the present work is related to the recent developments of 


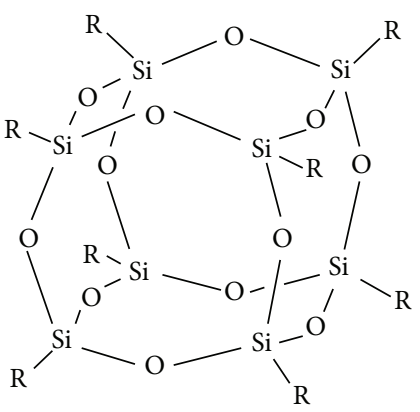

(a)

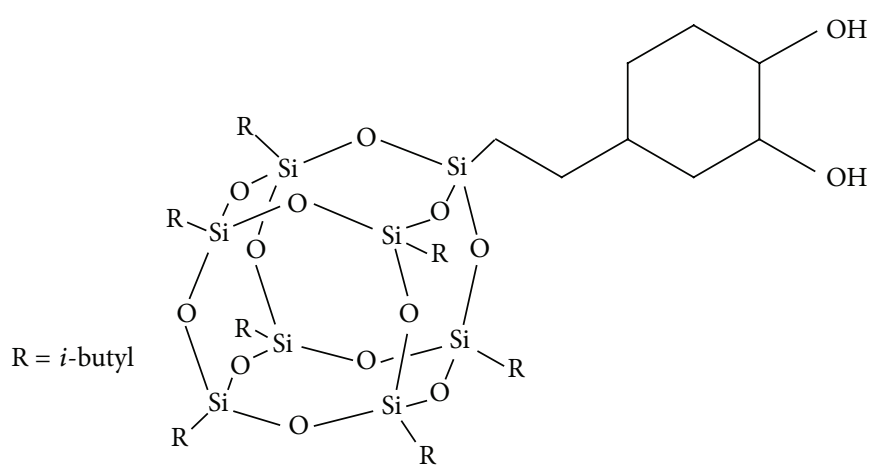

(b)

FIGURE 1: (a) Octaisobutyl POSS (oib-POSS) and (b) trans-cyclohexanediolisobutyl POSS (POSS-OH).

cyclic poly(butyleneterephthalate) (c-PBT) oligomers. Since the pioneer work of Brunelle et al. [25], who demonstrated that the polymerization of cyclic oligomers, with a variety of initiators, can be completed within minutes, the above monomer systems have been used in the development of composites and nanocomposites. In particular, as far as nanostructured system preparation is concerned, in situ polymerization of organically modified clay dispersed in low viscosity c-PBT allowed obtaining PBT-based nanocomposites, characterized by a high level of polymer intercalation [26-29]. Baets et al. [30] described the influence of the presence of multiwalled carbon nanotubes (MWCNT) in cPBT on the final polymer mechanical properties. Moreover, MWCNT were also functionalized with PBT, which was covalently attached onto the carbon nanotube surface, by a method based on in situ polymerization of c-PBT using a MWCNT-supported initiator. More recently, Fabbri et al. [31] prepared PBT/graphene composites by in situ polymerization of c-PBT in the presence of graphene, which turned out to be electrically conductive. Among the various nanostructured systems, developed by using c-PBT, also PBT/silica nanocomposites were prepared by using in situ polymerization [32]. Indeed, the incorporation of silica nanoparticles into c-PBT was found to affect both the properties of c-PBT resins and the final features of the nanostructured polymer. On this basis, the present work reports on novel nanostructured nanofibers prepared by electrospinning PBT/POSS hybrids, which were synthesized, for the first time, from c-PBT. In particular, a hydroxyl-bearing silsesquioxane, potentially capable of taking part to the ring-opening polymerization (ROP) of c-PBT, has been dissolved in the molten monomer system and the in situ polymerization has been carried out. The so-prepared hybrid systems have been electrospun in order to obtain nanostructured nanofibers.

\section{Experimental}

2.1. Materials. Cyclic oligomers of poly(butylene terephthalate) (c-PBT) were kindly supplied by Cyclics Corp.

Octaisobutyl POSS (referred to as oib-POSS in the following) and trans-cyclohexanediolisobutyl POSS (referred to as POSS-OH in the following) were purchased from Hybrid Plastics (USA) as crystalline powders and used as received. Chemical structures for oib-POSS $(M=873.6 \mathrm{~g} / \mathrm{mol})$ and POSS-OH $(M=959.7 \mathrm{~g} / \mathrm{mol})$ are reported in Figure 1.

Butyltin chloride dihydroxide was purchased from Sigma Aldrich and used as received.

2.2. POSS-Based Hybrid System Preparation. Before accomplishing the hybrid preparation, c-PBT was dried overnight at $80^{\circ} \mathrm{C}$. c-PBT was added to the glass reactor, namely, a laboratory internal mixer provided with a mechanical stirrer (Heidolph, type RZR1) which was connected to a vacuum line and evacuated for $30 \mathrm{~min}$ at $80^{\circ} \mathrm{C}$. Then, the reactor was purged with helium for $30 \mathrm{~min}$. The above operations were repeated at least three times to be sure to prevent humidity contact with the reagents. The reactor was placed in an oil bath at $190^{\circ} \mathrm{C}$ and when the monomer was completely molten, POSS and the catalyst were added under inert atmosphere. c-PBT/POSS systems were prepared by adding to the reaction mixture, both octaisobutyl and trans-cyclohexanediolisobutyl POSS, at various concentrations, from 2 to $10 \mathrm{wt} . \%$, by using a polymerization time of 10 minutes. Neat PBT was prepared and characterized under the same conditions, as reference material. Materials are identified in the text with the format polymer/POSS type (concentration), es.: PBT/POSS-OH(10). In order to evaluate the reaction yield after melt blending, all solid samples were broken into small pieces and purified from unreacted POSS by Soxhlet extraction with tetrahydrofuran (THF) for $48 \mathrm{~h}$. The grafting yield was calculated by weighing composite samples before and after the above treatment.

2.3. Electrospun Fiber Preparation. Polymeric solutions were prepared by dissolving $15 \mathrm{wt} . \%$ of PBT or PBT/POSS in the solvent mixture methylene chloride (MC, from Aldrich) and trifluoroacetic acid (TFA, from Aldrich) with a $1: 1 \mathrm{v} / \mathrm{v}$ ratio. The solutions were stirred for $6 \mathrm{~h}$ at room temperature to reach complete PBT dissolution. Electrospun nanofibers were prepared by using a conventional electrospinning system [10]. The viscous fluid was loaded into a syringe (Model Z314544, diameter $d=11.6 \mathrm{~mm}$, Aldrich Fortuna Optima) placed in the horizontal direction. A syringe pump (Harvard 
TABLE 1: Characteristics of the samples prepared.

\begin{tabular}{lcccc}
\hline Sample code & POSS type & POSS conc. (wt.\%) & Reaction yield (\%) & $M_{v} \cdot 10^{4}$ \\
\hline PBT & - & 0 & 99 & 99 \\
PBT(POSS-OH2) & POSS-OH & 2 & 99 & 1.9 \\
PBT(POSS-OH5) & POSS-OH & 5 & 58 & 1.7 \\
PBT(POSS-OH10) & POSS-OH & 10 & 9.5 & 2.1 \\
PBT(oib-POSS5) & oib-POSS & 5 & & 94 \\
\hline
\end{tabular}

Apparatus Model 44 Programmable Syringe Pump) was used to feed the needle with a controlled flow rate of $0.003 \mathrm{~mL} / \mathrm{min}$. The needle of the syringe (diameter $d=0.45 \mathrm{~mm}$ ) was connected to the positive electrode of Gamma High Voltage Research Power Supply (Model ES30P-5W) which generated a constant voltage. The negative electrode was attached to the grounded collector, an aluminium sheet wrapped on a glass cylinder (height $4 \mathrm{~cm}$, diameter $14.5 \mathrm{~cm}$ ). The needle tip and the ground electrode were incorporated into a plastic hollow cylinder (height $30.5 \mathrm{~cm}$, inner diameter $24 \mathrm{~cm}$, and thickness $3.5 \mathrm{~mm}$ ) chamber, internally coated with a polytetrafluoroethylene sheet (thickness $1 \mathrm{~mm}$ ), which was supplied with an XS Instruments digital thermohygrometer (Model UR100, accuracy $\pm 3 \% \mathrm{RH}$ and $\pm 0.8^{\circ} \mathrm{C}$ ) as humidity and temperature sensor to monitor and control the ambient parameters (temperature around $21^{\circ} \mathrm{C}$ ). A glass Brooks rotameter was used to keep the air flow $\left(F_{a}\right)$ constant in the enclosed electrospinning space. The air flow was fed into the chamber at atmospheric pressure from an inlet placed behind the collector.

2.4. Characterization. The intrinsic viscosity of the samples, dissolved in $0.5 \mathrm{~g} / \mathrm{dL}$ concentrated mixture solvent of phenol/1,1,2,2-tetrachloroethane $(\mathrm{w} / \mathrm{w}=60: 40)$, was determined with an Ubbelohde viscometer thermostated at $30 \pm$ $0.5^{\circ} \mathrm{C}$ in a water bath. $M_{v}$ was obtained from the following Mark-Houwink equation [33]:

$$
[\eta]=1.166 \times 10^{-4} M_{v}^{0.871} .
$$

${ }^{1} \mathrm{H}-\mathrm{NMR}$ spectra were obtained on a Varian $300 \mathrm{NMR}$, dissolving the samples in $\mathrm{CDCl}_{3}$. The ${ }^{1} \mathrm{H}-\mathrm{NMR}$ spectra were referenced to the residual solvent protons at ca. $7.26 \mathrm{ppm}$.

Differential scanning calorimetry (DSC) was performed under a continuous nitrogen purge on a Mettler calorimeter, Model TC10A. Both calibrations of heat flow and temperature were based on a run in which one standard sample (indium) was heated through its melting point. Samples having a mass between 5 and $11 \mathrm{mg}$ were used. The procedure was as follows: first heating scan at $10^{\circ} \mathrm{C} / \mathrm{min}$ from $25^{\circ} \mathrm{C}$ up to $250^{\circ} \mathrm{C}$, then cooling at $10^{\circ} \mathrm{C} / \mathrm{min}$ down to $25^{\circ} \mathrm{C}$, and, finally, second heating scan from $25^{\circ} \mathrm{C}$ to $250^{\circ} \mathrm{C}$, again at $10^{\circ} \mathrm{C} / \mathrm{min}$. The first scan was meant to erase the prior uncontrolled thermal history of the samples. The degree of crystallinity was determined by considering the melting enthalpy of $142 \mathrm{~J} / \mathrm{g}$ for $100 \%$ crystalline PBT [34]. Both temperature and heat flow were previously calibrated using a standard indium sample. To study the electrospun samples surface morphology, a Leica Stereoscan 440 scanning electron microscope was used. All the samples were thinly sputter-coated with carbon using a Polaron E5100 sputter coater. The fibers diameter and their distribution were measured using an image analyser, namely, ImageJ 1.41 software.

\section{Results and Discussion}

3.1. Preparation and Characterization of Hybrid Systems. The preparation of PBT/POSS hybrid systems, starting from cPBT, has been carried out by introducing into the reaction medium two different silsesquioxane molecules, a potentially reactive one (POSS-OH) and a nonreactive one (oib-POSS). Apart from their functional groups, the two POSS molecules differ also for the melting temperature $\left(T_{m}\right)$, being $T_{m}$ of POSS-OH $c a .140^{\circ} \mathrm{C}$ and that of oib-POSS $c a .250^{\circ} \mathrm{C}$. As such, it should be taken into account that also the above feature could lead to different behaviors of the two silsesquioxanes during the polymerization process, as POSS-OH turns out to be molten at the polymerization temperature $\left(190^{\circ} \mathrm{C}\right)$. Table 1 shows the reaction yields, calculated by extraction with THF, which is capable of solubilizing silsesquioxane molecules, the monomer, and the oligomers.

According to Table 1, it comes out that by using a catalyst concentration of $2 \mathrm{wt} . \%$ it was possible to reach a yield close to $100 \%$. It is of outmost relevance that the limited polymerization time applied, namely, 10 minutes, makes the process potentially applicable also to a reactive extrusion polymerization. Concentrations of 2 and $5 \mathrm{wt} \%$ of POSS$\mathrm{OH}$ in the reaction mixture are found not to influence the reaction yield, which evidences both complete conversion of c-PBT and reactivity of POSS-OH in these conditions. On the other hand, a significantly lower reaction yield was obtained for PBT/POSS-OH(10) showing that high loading of POSS is detrimental for the polymerization of C-PBT. As for the case of the system based on isobutyl-POSS, PBT/oib-POSS(5), it is likely that the yield drop (down to $94 \%$ ) is due to complete extraction of the unreacted oib-POSS by the washing solvent, which evidences the efficiency of the experimental procedure for the extraction of unbound silsesquioxanes.

Molecular masses of POSS-based hybrids were found to decrease by increasing the hydroxyl-silsesquioxane concentration in the reaction mixture. In particular, in the case of PBT/POSS-OH(10), prepared by adding $10 \mathrm{wt} . \%$ of POSS to the molten C-PBT, the reduction of molecular mass is significant, the sample being characterized by a molecular mass of only 5000, compared to about 21000 for pristine PBT. This finding highlights the active role of the hydroxylsilsesquioxane in the polymerization reaction. Conversely, isobutyl-POSS seems not to influence the characteristics of 


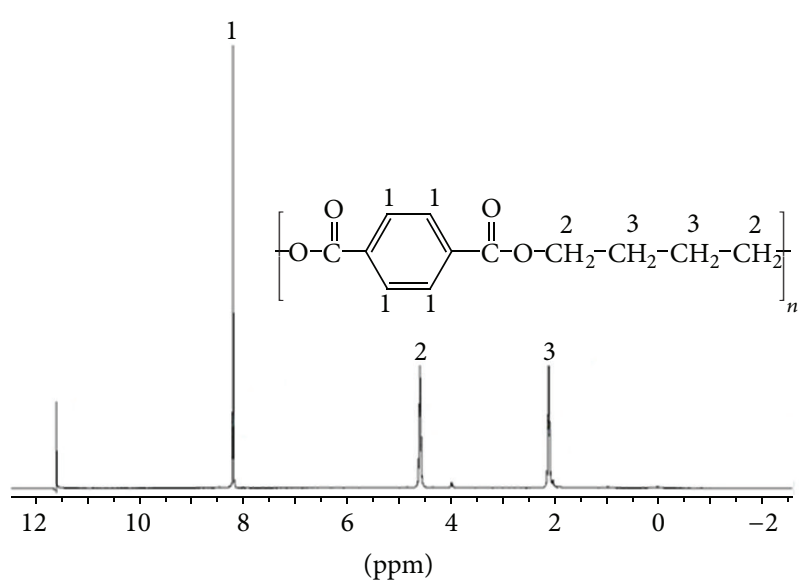

(a)

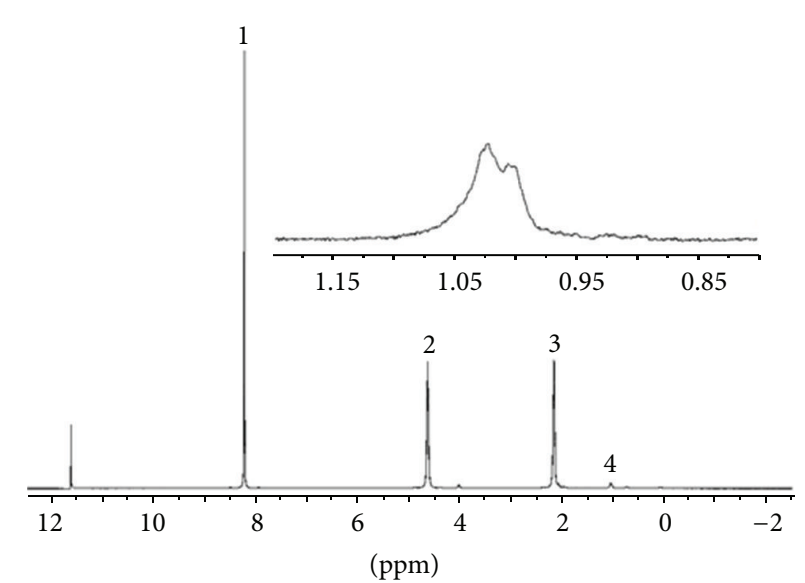

(b)

Figure 2: ${ }^{1} \mathrm{H}-\mathrm{NMR}$ spectra of (a) PBT(1) and (b) PBT(POSS-OH5).

TABLE 2: Thermal properties of the samples prepared.

\begin{tabular}{lcccccc}
\hline Sample code & POSS type & POSS conc. $($ wt. $\%)$ & $T_{m \mathrm{I}}\left({ }^{\circ} \mathrm{C}\right)$ & $T_{m \mathrm{II}}\left({ }^{\circ} \mathrm{C}\right)$ & $\Delta H_{m}(\mathrm{~J} / \mathrm{g})$ & 58 \\
\hline PBT & - & 0 & 224 & 210 & $X_{c}(\%)$ \\
PBT(POSS-OH2) & POSS-OH & 2 & 223 & 215 & 41 \\
PBT(POSS-OH5) & POSS-OH & 5 & 224 & 204 & 61 & 59 \\
PBT(POSS-OH10) & POSS-OH & 10 & 224 & 215 & 43 \\
PBT(oib-POSS5) & oib-POSS & 5 & 223 & 212 & 61 \\
\hline
\end{tabular}

the system prepared, the molecular mass of PBT/oib-POSS(5) being equal to that of the neat polymer.

The structure of the synthesized systems has been investigated by ${ }^{1} \mathrm{H}-\mathrm{NMR}$ measurements. Figure 2 shows ${ }^{1} \mathrm{H}-\mathrm{NMR}$ spectra of neat PBT (PBT) and that of PBT/POSS-OH(5), both samples being synthesized by using the same conditions.

Peaks at $8.09 \mathrm{ppm}$ are due to aromatic protons (1); those at $4.41 \mathrm{ppm}$ are assigned to the methylene protons (2) attached to the -O- in the ester groups and those at $2.19 \mathrm{ppm}$ to the unshielded methylene protons (3) of the PBT unit.

In the case of the PBT/POSS-OH(5) sample, based on $5 \mathrm{wt} . \%$ of POSS-OH, the general structure of the polymer seems not to change, but a new peak appears at $c a .1 \mathrm{ppm}$. This signal is due to the presence of POSS moiety, the same peak being present also in the spectrum of the neat silsesquioxane [35]. This finding confirms that POSS-OH is indeed chemically bound to PBT chains, the unreacted silsesquioxane having been removed by the purification process. On the basis of the previous described results and taking into account the information gathered by ${ }^{1} \mathrm{H}-\mathrm{NMR}$ measurements, it is possible to infer that the polymerization proceeds via a coordination-insertion mechanism, as described in the reaction mechanism reported in Figure 3.

The first step of the proposed mechanism consists of the coordination of both the monomer and of a silsesquioxane molecule to the Lewis-acid Sn metal center. One of the hydroxyl groups of the silsesquioxane subsequently attacks the carbonyl carbon of the monomer, followed by ring opening via acyl-oxygen cleavage, which ultimately results in the insertion of a c-PBT into the $\mathrm{O}-\mathrm{H}$ bond of the coordinated POSS. Clearly, the direct insertion of the silsesquioxane molecule into the polymer backbone indicates that POSS$\mathrm{OH}$, acting as an initiator, allows the obtainment of a hybrid system. As a consequence of this, by increasing the concentration of silsesquioxane in the reaction mixture, the number of initiated PBT chains increases, resulting in a lower average molecular mass.

The thermal properties obtained for the different formulation prepared are collected in Table 2. Neat PBT exhibits a double melting peak, which consists of a small peak at $210^{\circ} \mathrm{C}$ and a major one at $224^{\circ} \mathrm{C}$. Indeed, multiple peaks, which are typical for polyesters, including $\mathrm{PBT}$ prepared from c-PBT, are attributed to melting and recrystallization processes of thinner and less perfect crystallites into thicker and more perfect crystalline structures with a subsequent higher melting temperature [36-38]. As far as POSS-based hybrids are concerned, similar melting peaks are observed. The presence of the silsesquioxane molecule at the chain end seems not to affect the material thermal properties, since, for the imposed thermal history, the overall crystallinity as well as the maximum achievable crystal thickness and order is almost the same for all samples.

The sample PBT(POSS-OH10) deserves a particular comment as its low molecular mass is not associated with an increase of the crystallinity as expected. However, it is necessary to consider also the influence of the presence of POSS, 


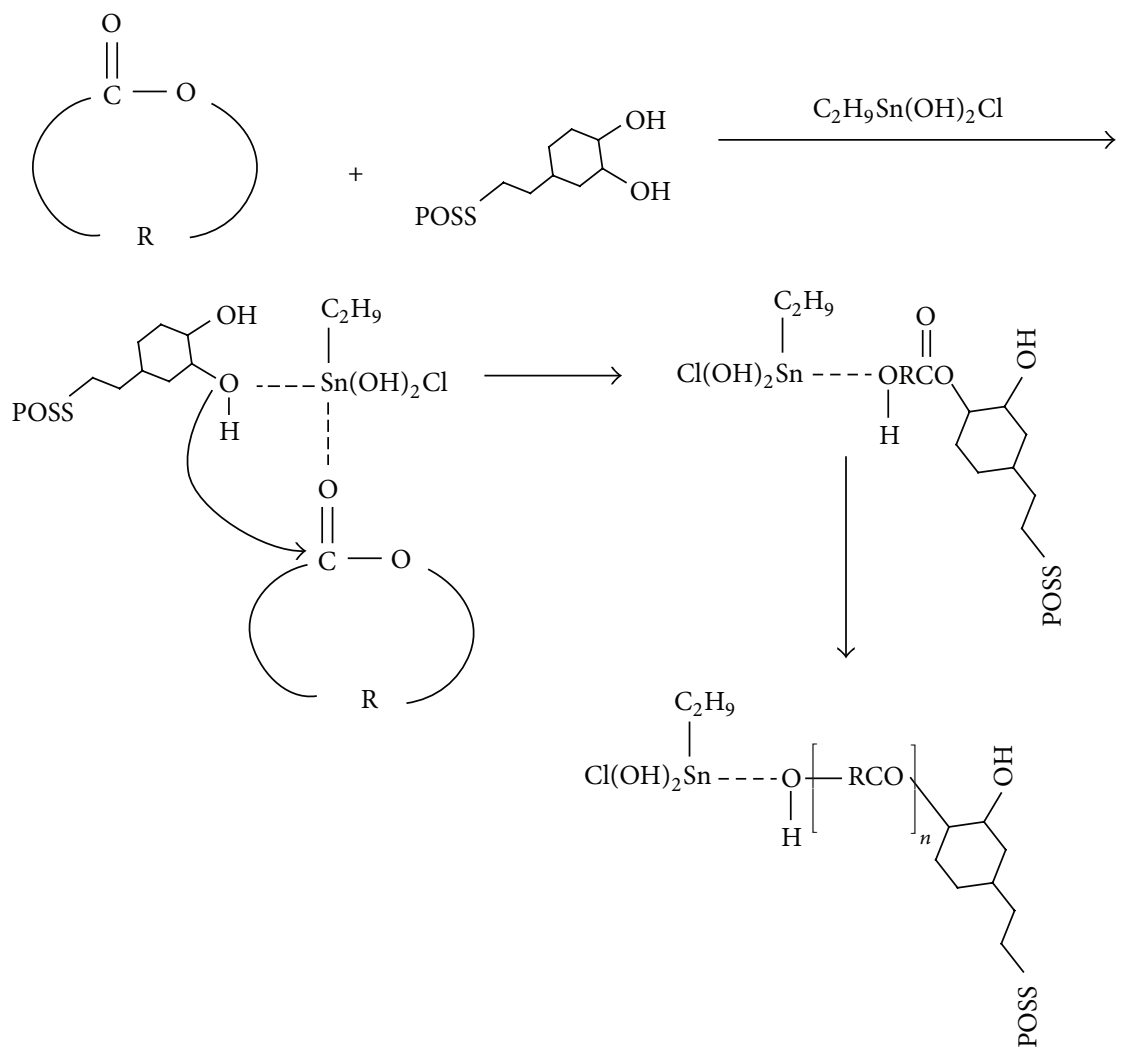

FIGURE 3: Proposed polymerization mechanism of c-PBT and POSS-OH.

which being directly linked to the macromolecular chain might limit the polymer organization, as already reported with other polymer matrices [39].

\subsection{Preparation and Characterization of Nanostructured Na-} nofibers. Both solutions PBT/POSS-OH hybrids and neat PBT, prepared from c-PBT, were electrospun by applying the same conditions (type of solvent, polymer concentration, voltage, humidity, etc.) used in a previous work [16]. Figure 4 compares a SEM micrograph of the nanofibers prepared from the PBT solution with those obtained by electrospinning the $\mathrm{PBT} / \mathrm{POSS}-\mathrm{OH}(5)$ solution. In the same figure, histograms of nanofiber diameter dimensions are reported.

Both mats are characterized by defect-free nanofibers, with no visible beads and with a similar average dimension, ca. $400 \mathrm{~nm}$. Nevertheless, by comparing the nanofiber diameter distribution, $\mathrm{PBT} / \mathrm{POSS}-\mathrm{OH}$ nanofibers exhibit narrower dimensional distribution than that for neat PBT. As the electrospinning conditions applied were the same for the preparation of both materials, this phenomenon has to be related to the modification of the polymer solution properties (polymer solubility, viscosity, surface tension, etc.) as a consequence of POSS insertion into PBT chains. Indeed, a similar effect was already assessed in the case of electrospun PVDF nanofibers, prepared by adding POSS in the electrospinning solutions [12]. In that case, the phenomenon was ascribed to the silsesquioxane molecules, which, without influencing the solution viscosity or conductivity, favored the formation of uniform structures, by decreasing the system surface tension. As far as our hybrid systems are concerned, taking into account the limited difference in molecular weight, thus weakly affecting viscosity of the solution, a similar effect of POSS on surface tension may be hypothesized.

\section{Conclusions}

Novel hybrid systems based on poly(butyleneterephthalate) (PBT) and hydroxyl-bearing polyhedral oligomeric silsesquioxanes (POSS) were developed by using cyclic poly(butyleneterephthalate) oligomers (c-PBT) as monomer system. Indeed, the polymerization reaction was found to occur through a coordination-insertion mechanism where the silsesquioxane molecules, acting as initiators, remain attached to the polymer backbone. It is of outmost interest that the used catalyst, namely, butyltin chloride dihydroxide, allowed reaching a complete conversion by applying a polymerization time (10 minutes) which is close to processing time reachable in melt reactive extrusion processing. Complete yields for polymerization were obtained even in the presence of reactive POSS, for concentrations up to $5 \% \mathrm{wt}$, whereas molecular mass was found to be decreased, depending on the POSS concentration.

Among the possible applications of the prepared PBT/ POSS hybrid system, the possibility to obtain nanofibers by 


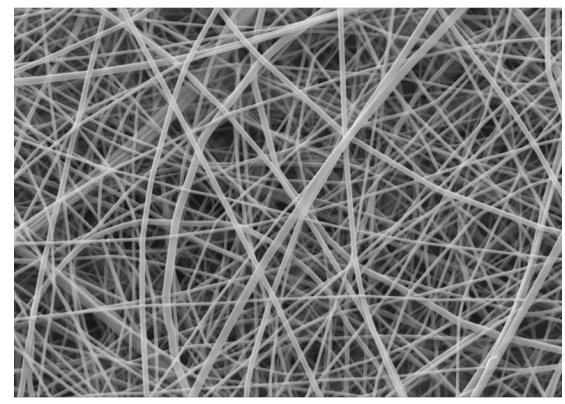
$100 \mathrm{~nm}$

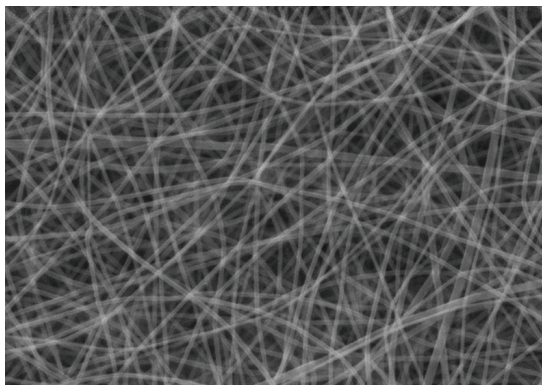

$100 \mathrm{~nm}$

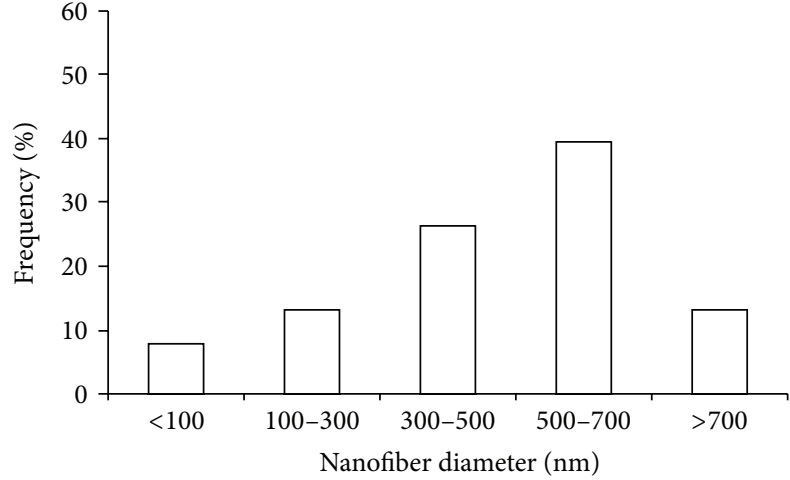

(a)

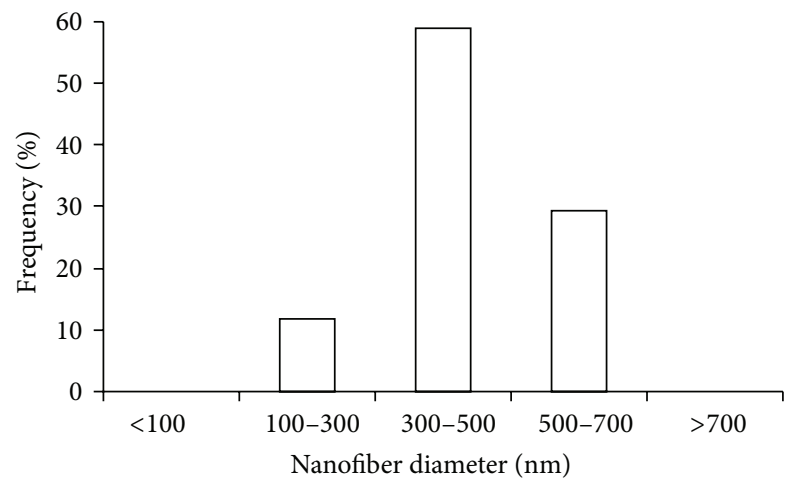

(b)

FIGURE 4: SEM micrograph of (a) PBT nanofibers and the relative diameter distribution; (b) PBT(POSS-OH5) nanofibers and the relative diameter distribution.

electrospinning was successfully assessed, showing defectfree fibers with an average diameter of $400 \mathrm{~nm}$. Furthermore, the nanostructured nanofibers were found to be more homogenous in diameter than those prepared starting from a neat PBT solution.

\section{Conflict of Interests}

The authors declare that there is no conflict of interests regarding the publication of this paper.

\section{Acknowledgment}

The authors are grateful to the Italian Ministry of Education and University for the 2010-2011 PRIN Project (Grant no. 2010XLLNM3_005).

\section{References}

[1] D. B. Cordes, P. D. Lickiss, and F. Rataboul, "Recent developments in the chemistry of cubic polyhedral oligosilsesquioxanes," Chemical Reviews, vol. 110, no. 4, pp. 2081-2173, 2010.

[2] O. Monticelli, A. Fina, E. S. Cozza, M. Prato, and V. Bruzzo, "POSS vapor phase grafting: a novel method to modify polymer films," Journal of Materials Chemistry, vol. 21, no. 44, pp. 1804918054, 2011.
[3] L. Valentini, S. B. Bon, O. Monticelli, and J. M. Kenny, "Deposition of amino-functionalized polyhedral oligomeric silsesquioxanes on graphene oxide sheets immobilized onto an amino-silane modified silicon surface," Journal of Materials Chemistry, vol. 22, no. 13, pp. 6213-6217, 2012.

[4] F. Baldi, F. Bignotti, L. Ricco, O. Monticelli, and T. Riccò, "Mechanical and structural characterization of POSS-modified polyamide 6," Journal of Applied Polymer Science, vol. 100, no. 4, pp. 3409-3414, 2006.

[5] I. Blanco, L. Abate, M. L. Antonelli, F. A. Bottino, and P. Bottino, "Phenyl hepta cyclopentyl-polyhedral oligomeric silsesquioxane (ph,hcp-POSS)/polystyrene (PS) nanocomposites: the influence of substituents in the phenyl group on the thermal stability," eXPRESS Polymer Letters, vol. 6, pp. 997-1006, 2012.

[6] F. Carniato, C. Bisio, G. Gatti et al., "Titanosilsesquioxanes embedded in synthetic clay as a hybrid material for polymer science," Angewandte Chemie-International Edition, vol. 48, no. 33, pp. 6059-6061, 2009.

[7] O. Monticelli, A. Fina, D. Cavallo, E. Gioffredi, and G. Delprato, "On a novel method to synthesize POSS-based hybrids: an example of the preparation of TPU based system," Express Polymer Letters, vol. 7, no. 12, pp. 966-973, 2013.

[8] A. Fina, H. C. L. Abbenhuis, D. Tabuani, and G. Camino, "Metal functionalized POSS as fire retardants in polypropylene," Polymer Degradation and Stability, vol. 91, no. 10, pp. 2275-2281, 2006.

[9] W.-Y. Chen, Y.-Z. Wang, S.-W. Kuo, C.-F. Huang, P.-H. Tung, and F.-C. Chang, "Thermal and dielectric properties and curing 
kinetics of nanomaterials formed from POSS-epoxy and metaphenylenediamine," Polymer, vol. 45, no. 20, pp. 6897-6908, 2004.

[10] N. Bhardwaj and S. C. Kundu, "Electrospinning: a fascinating fiber fabrication technique," Biotechnology Advances, vol. 28, no. 3, pp. 325-347, 2010.

[11] E. S. Cozza, O. Monticelli, and E. Marsano, "Electrospinning: A novel method to incorporate POSS into a polymer matrix," Macromolecular Materials and Engineering, vol. 295, no. 9, pp. 791-795, 2010.

[12] E. S. Cozza, O. Monticelli, O. Cavalleri, and E. Marsano, "Preparation, characterization, and properties of nanofibers based on poly(vinylidene fluoride) and polyhedral oligomeric silsesquioxane," Polymers for Advanced Technologies, vol. 23, no. 9, pp. 1252-1257, 2012.

[13] J. Choi, K. M. Lee, R. Wycisk, P. N. Pintauro, and P. T. Mather, "Sulfonated polysulfone/POSS nanofiber composite membranes for PEM fuel cells," Journal of the Electrochemical Society, vol. 157, no. 6, pp. B914-B919, 2010.

[14] K.-O. Kim, Y.-A. Seo, B.-S. Kim et al., “Transition behaviors and hybrid nanofibers of poly(vinyl alcohol) and polyethylene glycol-POSS telechelic blends," Colloid and Polymer Science, vol. 289, no. 8, pp. 863-870, 2011.

[15] J. Wang, A. Sutti, X. Wang, and T. Lin, "Fast responsive and morphologically robust thermo-responsive hydrogel nanofibres from poly( $N$-isopropylacrylamide) and POSS crosslinker," Soft Matter, vol. 7, no. 9, pp. 4364-4369, 2011.

[16] E. S. Cozza, Q. Ma, O. Monticelli, and P. Cebe, "Nanostructured nanofibers based on PBT and POSS: effect of POSS on the alignment and macromolecular orientation of the nanofibers," European Polymer Journal, vol. 49, no. 1, pp. 33-40, 2013.

[17] Y. Xue, H. Wang, D. Yu et al., "Superhydrophobic electrospun POSS-PMMA copolymer fibres with highly ordered nanofibrillar and surface structures," Chemical Communications, no. 42, pp. 6418-6420, 2009.

[18] E. S. Cozza, V. Bruzzo, F. Carniato, E. Marsano, and O. Monticelli, "On a novel catalytic system based on electrospun nanofibers and M-POSS," ACS Applied Materials \& Interfaces, vol. 4, no. 2, pp. 604-607, 2012.

[19] L. Gardella, A. Basso, M. Prato, and O. Monticelli, "PLA/POSS nanofibers: a novel system for the immobilization of metal nanoparticles," ACS Applied Materials \& Interfaces, vol. 5, no. 16, pp. 7688-7692, 2013.

[20] O. Monticelli, M. Putti, L. Gardella et al., "New stereocomplex PLA-based fibers: effect of POSS on polymer functionalization and properties," Macromolecules, vol. 47, no. 14, pp. 4718-4727, 2014.

[21] R. M. D. Soares, V. L. Patzer, R. Dersch, J. Wendorff, N. P. da Silveira, and P. Pranke, "A novel globular protein electrospun fiber mat with the addition of polysilsesquioxane," International Journal of Biological Macromolecules, vol. 49, no. 4, pp. 480-486, 2011.

[22] C.-K. Kim, B.-S. Kim, F. A. Sheikh, U.-S. Lee, M.-S. Khil, and H.Y. Kim, "Amphiphilic poly(vinyl alcohol) hybrids and electrospun nanofibers incorporating polyhedral oligosilsesquioxane," Macromolecules, vol. 40, no. 14, pp. 4823-4828, 2007.

[23] J. Wu, S. Hou, D. Ren, and P. T. Mather, "Antimicrobial properties of nanostructured hydrogel webs containing silver," Biomacromolecules, vol. 10, no. 9, pp. 2686-2693, 2009.

[24] M. Xue, X. Zhang, L. Ma et al., "Structure and thermal behavior of EPDM/POSS composite fibers prepared by electrospinning,"
Journal of Applied Polymer Science, vol. 128, no. 4, pp. 2395-2401, 2013.

[25] D. J. Brunelle, J. E. Bradt, J. Serth-Guzzo et al., "Semicrystalline polymers via ring-opening polymerization: preparation and polymerization of alkylene phthalate cyclic oligomers," Macromolecules, vol. 31, no. 15, pp. 4782-4790, 1998.

[26] A. R. Tripathy, E. Burgaz, S. N. Kukureka, and W. J. MacKnight, "Poly(butylene terephthalate) nanocomposites prepared by insitu polymerization," Macromolecules, vol. 36, no. 23, pp. 8593$8595,2003$.

[27] G. Lanciano, A. Greco, A. Maffezzoli, and L. Mascia, "Effects of thermal history in the ring opening polymerization of CBT and its mixtures with montmorillonite on the crystallization of the resulting poly(butylene terephthalate)," Thermochimica Acta, vol. 493, no. 1-2, pp. 61-67, 2009.

[28] F. Wu and G. Yang, "Poly(butylene terephthalate)/organoclay nanocomposites prepared by in-situ bulk polymerization with cyclic poly(butylene terephthalate)," Materials Letters, vol. 63, no. 20, pp. 1686-1688, 2009.

[29] C. Wan, F. Zhao, X. Bao, B. Kandasubramanian, and M. Duggan, "Surface characteristics of polyhedral oligomeric silsesquioxane modified clay and its application in polymerization of macrocyclic polyester oligomers," Journal of Physical Chemistry B, vol. 112, no. 38, pp. 11915-11922, 2008.

[30] J. Baets, A. Godara, J. Devaux, and I. Verpoest, “Toughening of polymerized cyclic butylene terephthalate with carbon nanotubes for use in composites," Composites Part A: Applied Science and Manufacturing, vol. 39, no. 11, pp. 1756-1761, 2008.

[31] P. Fabbri, E. Bassoli, S. B. Bon, and L. Valentini, "Preparation and characterization of poly (butylene terephthalate)/graphene composites by in-situ polymerization of cyclic butylene terephthalate," Polymer, vol. 53, no. 4, pp. 897-902, 2012.

[32] Z. Jiang, S. Siengchin, L. Zhou, M. Steeg, J. Karger-Kocsis, and H. C. Man, "Poly (butylene terephthalate)/silica nanocomposites prepared from cyclic butylene terephthalate," Composites Part A: Applied Science and Manufacturing, vol. 40, no. 3, pp. 273-278, 2009.

[33] F. Wu and G. Yang, "Synthesis and properties of poly(butylene terephthalate)/multiwalled carbon nanotube nanocomposites prepared by in situ polymerization and in situ compatibilization," Journal of Applied Polymer Science, vol. 118, no. 5, pp. 2929-2938, 2010.

[34] H. Parton, J. Baets, P. Lipnik, B. Goderis, J. Devaux, and I. Verpoest, "Properties of poly(butylene terephthatlate) polymerized from cyclic oligomers and its composites," Polymer, vol. 46, no. 23, pp. 9871-9880, 2005.

[35] O. Monticelli, D. Cavallo, S. Bocchini, A. Frache, F. Carniato, and A. Tonelotto, "A novel use of Ti-POSS as initiator of L-lactide ring-opening polymerization," Journal of Polymer Science, Part A: Polymer Chemistry, vol. 49, no. 22, pp. 47944799, 2011.

[36] M. C. Righetti and A. Munari, "Influence of branching on melting behavior and isothermal crystallization of poly(butylene terephthalate)," Macromolecular Chemistry and Physics, vol. 198, no. 2, pp. 363-378, 1997.

[37] Y. Liu, Y.-F. Wang, T. G. Gerasimov, K. H. Heffner, and J. P. Harmon, "Thermal analysis of novel underfill materials with optimum processing characteristics," Journal of Applied Polymer Science, vol. 98, no. 3, pp. 1300-1307, 2005.

[38] Z. A. M. Ishak, K. G. Gatos, and J. Karger-Kocsis, "On the insitu polymerization of cyclic butylene terephthalate oligomers: 
DSC and rheological studies," Polymer Engineering \& Science, vol. 46, no. 6, pp. 743-750, 2006.

[39] L. Zheng, A. J. Waddon, R. J. Farris, and E. B. Coughlin, "X-ray characterizations of polyethylene polyhedral oligomeric silsesquioxane copolymers," Macromolecules, vol. 35, no. 6, pp. 2375-2379, 2002. 

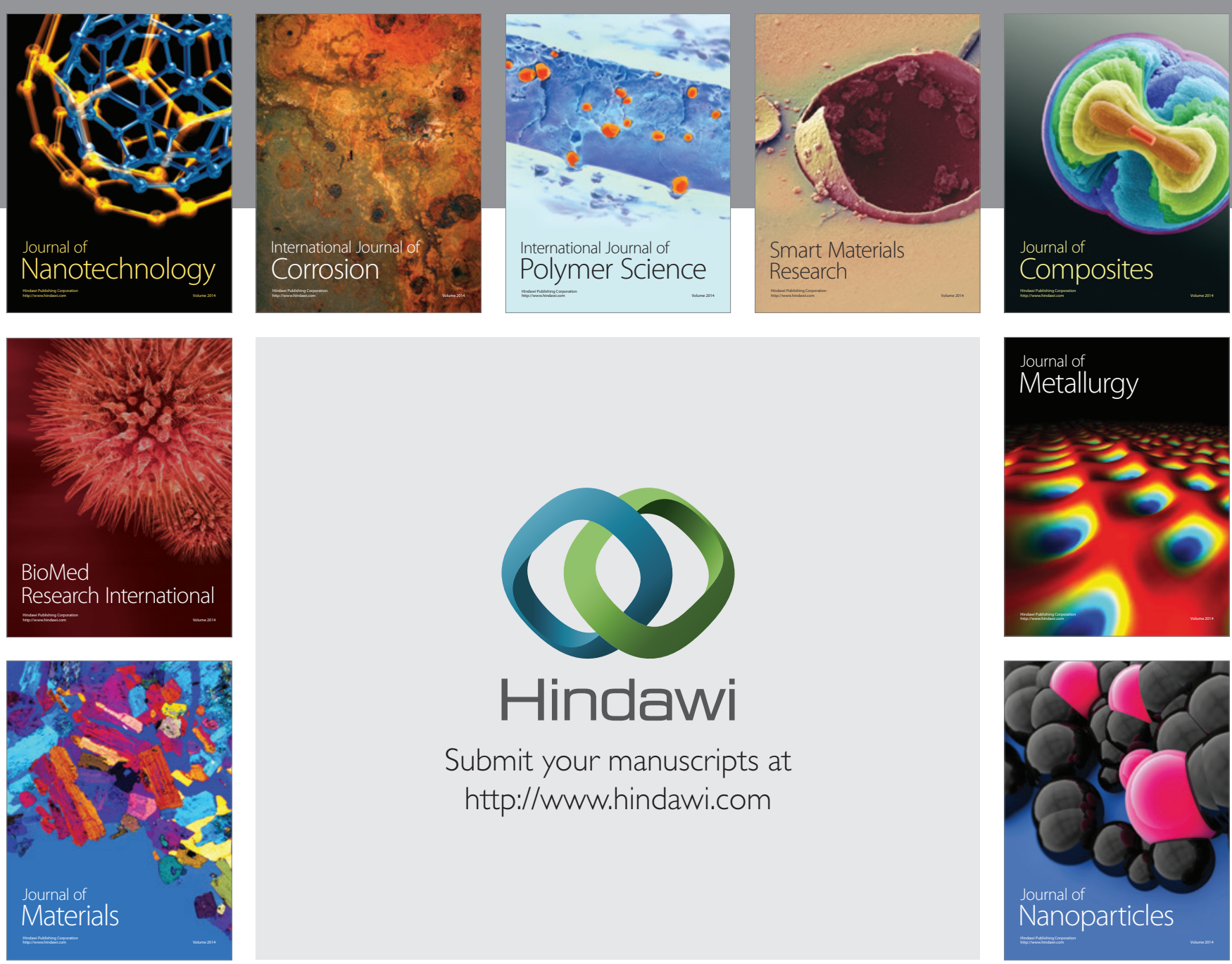

Submit your manuscripts at http://www.hindawi.com
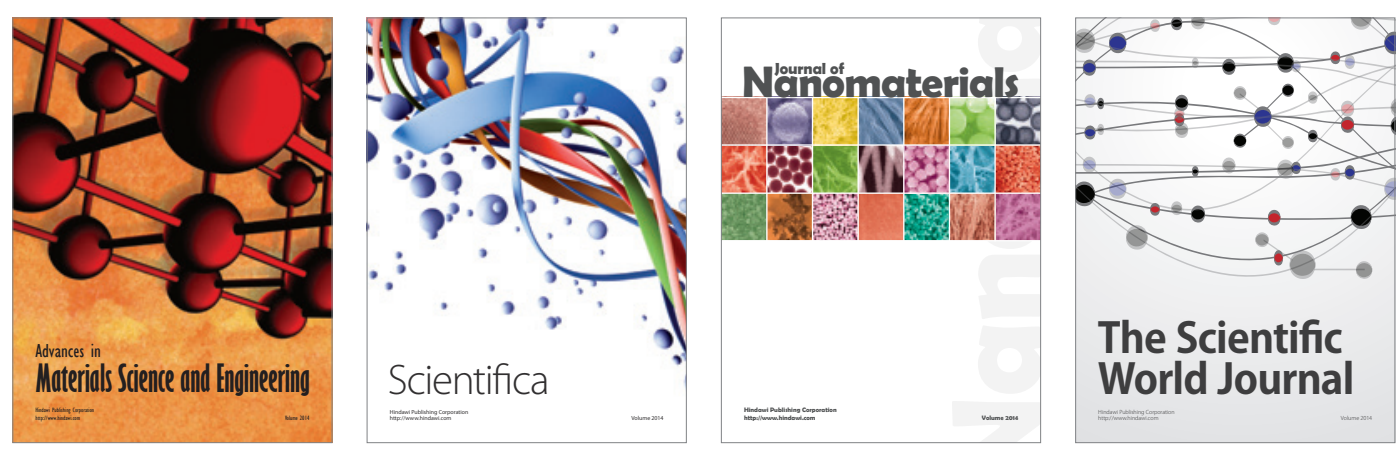

\section{The Scientific World Journal}
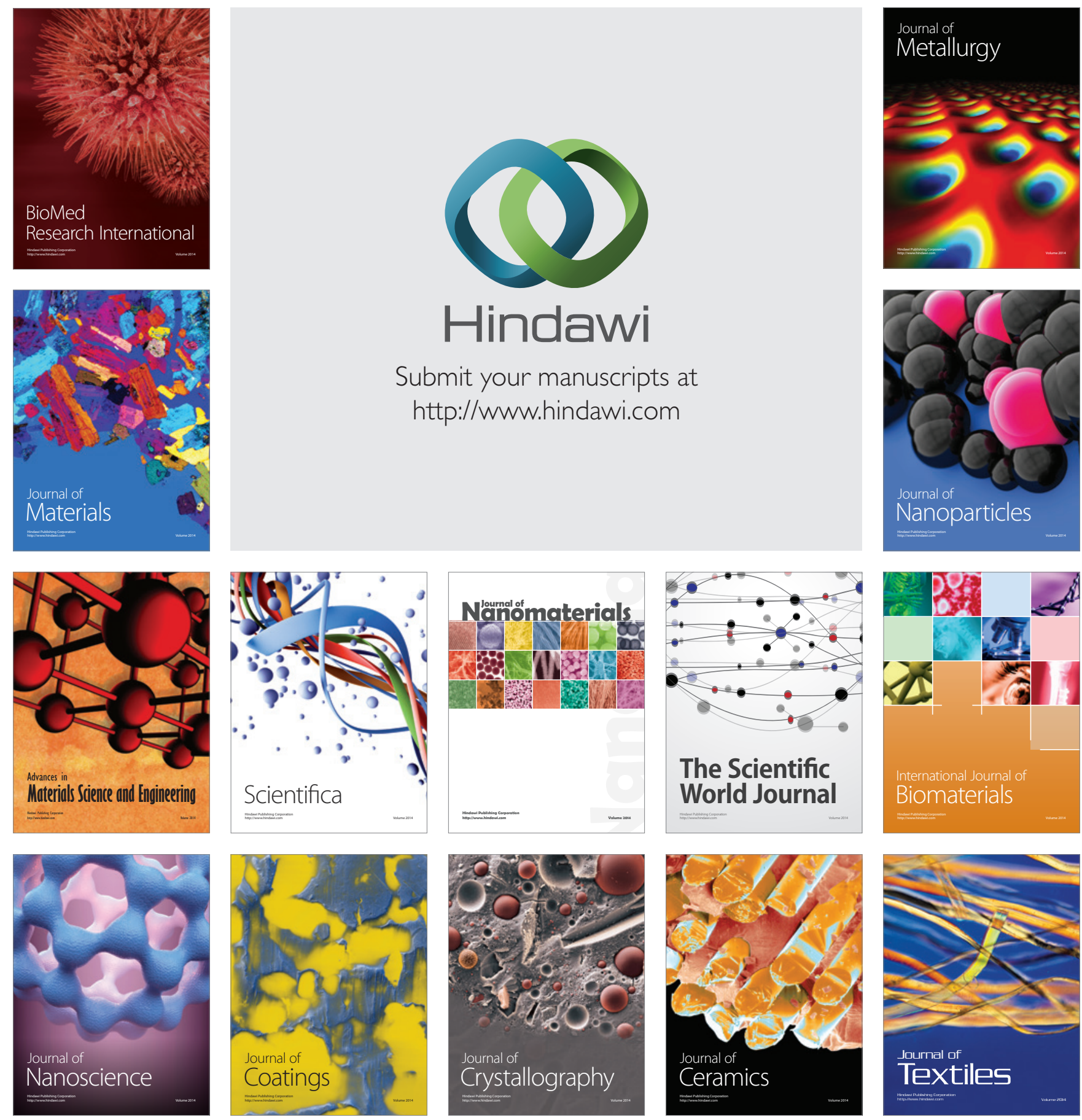